\title{
Bemestar animal e dor - uma revisão sobre mudanças comportamentais em bovinos
}

\author{
Ollé, M.A. ${ }^{@} ;$ Oliveira, C.A.; Lopes, L.S.; Barbosa, A.A.; Feijó, J.O., Rabassa, V.R.; Corrêa, M.N.; Brauner, C.C. e Del Pino, \\ F.A.B.
}

Núcleo de Pesquisa, Ensino e Extensão em Pecuária - Universidade Federal de Pelotas. Pelotas-RS. Brasil

PalaVRas chaVe adicionals

Escalas de dor.

Métodos observacionais.

Produtividade.

Saúde animal.

\section{RESUMO}

Ao longo de décadas a pecuária brasileira experimentou um grande desenvolvimento nas áreas de nutrição, genética, manejo e em especial no que tange o bemestar animal (BEA). Um fator que está inversamente relacionada ao BEA é a dor, no entanto a mensuração da dor ainda é um desafio para os produtores e pesquisadores, visto que os bovinos são estoicos e não demonstram sentimentos. Apesar disso, tem-se que a dor promove na saúde do animal diversos transtornos e como consequência pode ocasionar em uma experiência sensorial e emocional desagradável associada a injúria atual ou potencial. Dessa forma, o objetivo dessa revisão é apresentar os avanços e a importância de avaliar animais acometidos por algum tipo de injuria através de métodos e técnicas de identificação e mensuração da dor, além de sua possível influência no comportamento e bemestar dos bovinos. Estudos nesse âmbito utilizam análises fisiológicas, hormonais e metabólicas, bem como marcadores específicos para dor, aliados a técnica de observação comportamental. Todavia não existe um método que leve em consideração a união de todos esses fatores para bovinos, indicando assim o quão importante é a busca por novos meios de diagnóstico que possam ser aplicados no campo. Um desses avanços se dá através de novas tecnologias para avaliar o comportamento animal, o qual prediz algum tipo de desconforto. Conclui-se que os avanços nesse quesito são constantes e têm sido realizadas através da avaliação comportamental, afim de promover qualidade e segurança dos produtos e consequentemente melhorar a produtividade, sustentabilidade e lucratividade do sistema produtivo.

\section{Animal welfare and pain - a review on behavioural changes in cattle}

\section{SUMMARY}

\section{ADDITIONAL KEY WORDS}

Animal health.

Observational methods.

Pain scales.

Productivity.

INFORMATION

Cronología del artículo.

Recibido/Received: 07.09.2020

Aceptado/Accepted: 23.03 .2021

On-line: 15.04 .2021

Correspondencia a los autores/Contact e-mail:

obmorenikeji@futa.edu.ng
Over decades, Brazilian livestock has experienced a great development in the areas of nutrition, genetics, management and especially with regard to animal welfare. A factor that is inversely related to welfare is pain, however pain measurement is still a challenge for producers and researchers, since cattle are stoic and do not show feelings. Despite this, it is known that pain promotes various disorders in the animal's health and, as a consequence, can cause an unpleasant sensory and emotional experience associated with current or potential injury. Thus, the objective of this review is to present the advances and the importance of evaluating animals affected by some type of injury through methods and techniques for identifying and measuring pain, in addition to its possible influence on the behavior and welfare of cattle. Studies in this field use physiological, hormonal and metabolic analyzes, as well as specific markers for pain, combined with the behavioral observation technique. However, there is no method that takes into account the combination of all these factors for cattle, thus indicating how important is the search for new means of diagnosis that can be applied in the field. One of these advances is through new technologies to assess animal behavior or what is the type of discomfort. Conclude that the advances in this case are constant and those that were made during the behavioral evaluation, in order to promote quality and safety of the products and consequently improve the recovery, sustainability and profitability of the productive system.

\section{INTRODUÇÃO}

A pecuária leiteira teve significativos avanços nas áreas de nutrição, genética e manejo com grande inserção de modelos intensivos de criação nos sistemas produtivos. Tais mudanças culminaram ganhos econômicos e socias importantes, porém nem sempre o bem-estar tem sido levado em conta, todavia é sabido que medidas de conforto são fundamentais tanto ao ponto de vista de qualidade de vida quanto produtivo (Barkema et al., 2015).

Produtos oriundos de sistemas com alto grau de bem-estar animal apresentam valores agregados de ordem econômica e ética atendendo a uma demanda atual de mercado (Bond et al., 2012). Tal tema provavelmente surge do crescente interesse dos consumidores pela qualidade e segurança dos produtos que 
consomem. Essa preocupação cresce paralelamente ao desenvolvimento socioeconômico, alterando o perfil dos consumidores (Rocha et al., 2008), os quais demandam por manejos que atendam as normas de criação propiciando o máximo de conforto e bem-estar a esses animais. Assim, à medida que a sociedade reconhece $\mathrm{o}$ sofrimento animal como um fator relevante, o bem-estar promove destacado valor econômico aos sistemas produtivos (Molento, 2005; Silva et al., 2011).

Um fator inversamente associado ao bem-estar animal (BEA) é a dor, no qual reproduz para a saúde do animal múltiplos transtornos, como também pode estabelecer uma experiência sensorial e emocional desagradável associada a injúria atual ou potencial (Silva et al., 2011). Além disso, é considerada um potencial ativador das respostas neuroendócrinas, que dispõe o indivíduo a mudanças fisiológicas e comportamentais. Dessa maneira, alterações fisiológicas, comportamentais e emocionais são indicadores que permitem, em conjunto, o reconhecimento e classificação do grau de dor (Aleixo et al., 2016).

A identificação da dor em ruminantes, ao longo da evolução da pecuária tornou-se peça chave para o bom desempenho do sistema, muito em decorrência dos próprios consumidores por mudarem seus hábitos de consumo, optando por produtos em que os animais foram assegurados de BEA. Devendo assim, a dor ser identificada, mensurada e contida. Apesar disso, ainda existem poucos estudos com testes efetivos para identificação da dor nas diversas espécie (Hoag \& Lemme, 2018).

O reconhecimento da dor e seu alívio é pertinente para o BEA e são notórios os progressos nessa área através do desenvolvimento de pesquisas com escore de dor, escalas baseadas em comportamentos ou expressões faciais, além de avaliações comportamentais através de dispositivos eletrônicos. Entretanto, muito dessas avaliações são especificas para cada tipo de lesão, doença e procedimentos dolorosos. Sabe-se que alguns estudos utilizam análises fisiológicas, hormonais e metabólicas, bem como marcadores específicos para dor, aliado a técnica de observação comportamental. Todavia não existe um método que leve em consideração a união de todos esses fatores para bovinos, indicando assim, o quão importante é a busca por novos meios de diagnóstico que possam ser aplicados no campo (Amaral et al., 2017). Além de métodos persuasivos da avaliação de dor em ruminantes que englobem os mecanismos de proteção do corpo e manutenção da vida. Sendo assim, o objetivo deste trabalho é apresentar uma revisão sobre os avanços e a importância de avaliar animais acometidos por algum tipo de injuria, através de métodos e técnicas de identificação e mensuração da dor e, como esse pode influenciar o comportamento e o bem-estar dos bovinos.

\section{INFLUÊNCIA DA DOR NO BEM-ESTAR ANIMAL}

Segundo a Organização Mundial da Saúde Animal (OIE, 2019), bem-estar animal (BEA) nada mais é do que o estado físico e mental de um indivíduo em relação ao meio em que vive. Logo, o conceito de BEA está diretamente ligado às necessidades, liberdades, adaptações e saúde dos animais, tornando-se um termo multifatorial e com diferentes conceitos entre autores, mas que em suma visa qualidade de vida do indivíduo. Tem-se que um animal para estar em estado de BEA necessita encontrar-se confortável, nutrido, seguro, livre de doenças, dores, medo e angústia, desprendido para expressar seu comportamento biológico (Whay \& Shearer, 2017).

De acordo com Broom \& Molento (2004), o BEA é uma ciência indispensável aos profissionais que trabalham em torno da interação entre humanos e animais. Conforme os mesmos, os efeitos deletérios sobre o BEA podem ser oriundos de situações como: doenças, traumatismos, fome, manejo inadequados, entre outros. Um dos principais conceitos de produção animal é aquele que estabelece que a saúde e o bem-estar dos animais domésticos têm uma relação direta e indireta com a sua eficiência produtiva. Desta forma, os animais demonstram alto desempenho, em termos de produção de leite, quando estão com uma boa saúde e assim com altos padrões de bem-estar (Von Keyserlingk et al., 2009; Barkema et al., 2015).

O BEA é alicerçado em códigos morais e éticos, o que gera equilíbrio na produção animal através de produtores mais conscientes dos impactos negativos que os animais demonstram quando encontram-se fora do seu estado natural. Da mesma forma o mercado econômico deixam para traz a ideia de que os animais são apenas um recurso com valor usável. O conforto gerado a partir do conhecimento de que o animal foi tratado de forma apropriada para sociedade tem ganhado potencial econômico aos ramos alimentícios (Molento, 2005).

Um dos componentes fundamentais para um animal apresentar o BEA é a saúde e essa tem ligação ao ambiente onde vivem, principalmente em animais confinados, assim, o indivíduo pode apresentar-se sadio, contudo ainda pode demonstrar comportamentos anormais mostrando com isso que o ambiente não permitiu ao mesmo expressar seu comportamento natural (Broom \& Molento, 2004; Whay \& Shearer, 2017). Muitos consumidores associam a criação de animais a pasto com um adequado BEA (Von Keyserlingk et al., 2013), erroneamente, pois diversos estudos relatam que os animais podem não estar expressando as cinco liberdades citadas por FAWC (1993), independente do sistema de criação em que estão.

Outro principal componente ligado ao BEA e a saúde dos animais é a dor, principalmente em bovinos (Thomsen et al., 2010; Fajt et al., 2011; Molgaard et al., 2012), e esta encontra-se diretamente ligada a enfermidades que acometem os animais, tais como: claudicação (Bruijinis et al., 2013; Whay e Shearer, 2017), mastite (Proudfoot et al., 2014; Sepúlveda-Varas et al., 2016), metrite (Stojkov et al., 2015; Barragan et al., 2016; Stangaferro et al., 2016; Barragan et al., 2018; Neave et al., 2018), cetose (González et al., 2008), entre outros. No qual todos estes problemas de saúde em vacas leiteiras resultam em perdas de produção, levando a custos com tratamento (Bruijinis et al., 2013; Dittrich et al., 2019) e influenciando na lucratividade do produtor rural. 
A definição de dor foi descrita pela International Association for the Study of Pain, configura-se como a experiência emocional e sensitiva desagradável associada a uma lesão real ou potencial de um tecido (IASP, 1994). O que lhe torna uma experiência única e individual, alterada pelo conhecimento prévio de um dano que pode ser existente ou presumido (Trindade et al., 2013). A percepção da dor em animais e humanos é semelhante e ocorre por meio de múltiplos receptores de tecido projetados para detectar estímulos mecânicos, químicos e/ou térmicos. Esses receptores estão bem distribuídos por todo o corpo, permitindo a sensação de estímulos prazerosos e nocivos (Whay \& Shearer, 2017).

No entanto, a avaliação da dor é complexa, pois tanto a manifestação dolorosa quanto a conduta médica possuem caráter subjetivo (Viñuela-Fernández et al., 2007) e, por isso, pode ser difícil diagnosticá-la em animais devido também à falta de expressão verbal (Anil et al., 2002). Além disso a percepção do processo doloroso é uma experiência individual, e o quanto dessa experiência se traduz em um comportamento observável e mensurável depende de vários fatores tais como: espécie, sexo, peso corporal, condicionamento prévio, dominância e saúde em geral, além das condições do meio ambiente no momento da observação (Almeida et al., 2006; Klaumann et al., 2008). O que dificulta o diagnóstico e tornam ainda mais relevantes estudos específicos em cada espécie animal.

Os ruminantes possuem um limiar de dor alto, esse limiar é definido como a intensidade mínima do estímulo que é percebido como doloroso, ou seja, são menos sensíveis às dores que os cachorros, gatos e humanos, por exemplo (Silva et al., 2011; Trindade et al., 2013). O limiar de dor varia entre espécies e até em indivíduos da mesma espécie. De acordo com Nogueira et al. (2008), já foram comprovadas diferenças entre os sexos quanto ao impacto da condição dolorosa, comportamentos adaptativos à dor demonstrado que homens e mulheres diferem quanto à tolerância e sensibilidade à dor, não sendo diferente nos animais.

A avaliação da dor varia conforme o tipo da mesma. A dor aguda de uma lesão pode ser identificada imediatamente porque provoca manifestações de desconforto do animal à palpação da área acometida, corroborando, Stojkov et al. (2015) observaram que a inflamação associada à metrite é dolorosa e que a resposta à dor pode ser detectada durante a palpação retal com e sem palpação uterina, havendo mudança comportamental, como dorso arqueado, além de alteração fisiológicas. Ainda, o animal pode demonstrar dor mediante sinais clínicos explícitos, como tenesmo, disúria, disfagia, claudicação, vocalização e/ou inquietação. Já a dor de intensidade moderada pode ser menos evidente, causando alterações na posição e postura corporal, na alimentação, nos padrões de sono, na autolimpeza e na locomoção, dificultando a percepção durante observações de curto prazo (Erickson \& Kitchell, 1992; Hellebrekers, 2002).

Contudo, apesar do limiar de dor de vacas ser maior do que os demais animais domésticos, e com isso ser mais dificultoso sua mensuração fisiológica e com- portamental, não significa que os mesmos não sentem dor. Esse desafio é intensificado nos bovinos porque, como são presas em seu estado natural, podem evitar expressar dor para limitar a vulnerabilidade (Anil et al., 2002), comprometendo tanto a saúde, a eficiência produtiva, quanto o BEA.

Em geral, nos animais a dor só é tratada quando o profissional e o proprietário reconhecem a sua presença e julgam a sua gravidade (Viñuela-Fernández et al., 2007). Todavia, tendo a dor como um mecanismo fundamental de proteção do corpo e manutenção da vida, o entendimento da sua fisiologia, diagnóstico e tratamento em grandes animais, pode maximizar o BEA e reduzir os prejuízos em animais de produção (Silva et al., 2011).

Como os animais são incapazes de relatar sua dor, sua identificação requer a capacidade de entender o comportamento das espécies-alvo, sendo assim as mudanças de comportamento normalmente observadas nos animais com dor e as alterações específicas que ocorrem em cada animal, isto é, o comportamento do animal em resposta à dor (Anil et al., 2002).

Conforme Hulsen (2016), o comportamento sempre prediz que algo está errado, no entanto, o maior desafio é limitá-lo, identificá-lo precocemente e tomar medidas necessárias rapidamente. Geralmente esses sinais iniciam com animais do grupo de risco, por exemplo animais de alta produção, tornando esses animais os principais indicadores de rebanho. Apesar disso, vale destacar que a observação deve ser feita em todo o rebanho desde bezerras, novilhas, vacas secas até vacas lactantes.

Há diversas formas de identificar, mensurar e caracterizar a dor e, consequentemente o BEA, como por exemplo, utilizando indicadores fisiológicos, como frequência respiratória e cardíaca, temperatura e condição corporal, indicadores metabólicos, tais como cortisol, ácidos graxos não esterificados (AGNE) e proteínas de fase aguda, bem como indicadores comportamentais através de observações, sejam elas alterações na aparência e expressão facial (Gleerup et al. 2015; Amaral et al., 2017), social e alimentar (Broom \& Molento, 2004; Palmer et al., 2012; Barragan et al., 2018; Barker et al., 2018) e/ou através de escores específicos para determinação da dor, em muitos casos já fidelizados (Flower \& Weary, 2006; Dutton et al., 2008; Stojkov et al., 2015; Coetzee et al., 2017). Entretanto, existem limitações quanto aos indicadores comportamentais, visto o número crescente de vacas nos rebanhos dificultando as observações comportamentais visualmente e individualmente, o que implica em uma necessidade crescente de detecção automatizada de alterações desses padrões para identificar vacas que mostram "comportamento anormal" (Dittrich et al., 2019).

Assim, a utilização crescente de tecnologias na pecuária tem permitido que os produtores tenham acesso a fontes de dados que podem ajudar a melhorar ainda mais a saúde e o BEA (Barkema et al., 2015; Beauchemin, 2018). Portanto, a dor, a doença e outros fatores podem levar a mudanças comportamentais de vacas leiteiras, mas essas mudanças podem diferir dependendo da causa (Sepúlveda-Varas et al., 2016; Itle et al., 
2015; Molgaard et al., 2012; Dittrich et al., 2019). Além disso também interagem com fatores externos.

A Autoridade Europeia para a Segurança dos Alimentos (2012) recomenda sistemas de monitoramento para prevenir de forma eficaz as doenças de produção e garantir o BEA. Leblanc et al. (2006) provaram as vantagens de prevenir doenças antes que elas se tornassem clinicamente relevantes e, recomendam o monitoramento dos animais para verificar o estado de saúde do rebanho, visto que a chance de detectar precocemente alterações no estado de saúde do rebanho aumenta com o monitoramento regular do comportamento (Gruber \& Mansfeld, 2019) tornando a detecção precoce de doenças uma questão de bem-estar (Broom \& Fraser, 2015). Alterações como duração prolongada de ócio ou redução na duração da alimentação causada por distúrbios metabólicos ou infecções servem como ferramenta de detecção para indivíduos doentes (Dittrich et al., 2019).

Dessa forma, à medida que a sociedade e as técnicas experimentais evoluem, tornam-se importante as mensurações de dor sobre o grau de BEA, pois é notório que ao longo dos anos e com o surgimento de novos métodos de criação animal baseados na sustentabilidade ambiental o valor econômico em adotar esse conceito pelas indústrias e propriedades refere-se diretamente a bons níveis de produtividade e lucratividade (Bond et al., 2012).

\section{INDICADORES COMPORTAMENTAIS, FISIOLÓGICOS E BIOMARCADORES DA DOR}

A principal ferramenta usada para avaliar a dor em animais é o comportamento específico da espécie (Flecknell, 2008). Basta os produtores e profissionais observarem e saberem ler os sinais que a vaca demonstrará seu estado de saúde e bem-estar através de mudanças no comportamento, postura e características físicas. Todo o comportamento tem uma razão e explicação, seja para satisfazer suas necessidades, reação a um estímulo ou uma urgência física causada por dor, doença, parto ou hormônio. Dessa forma, o ideal é se perguntar "por que a vaca está fazendo isso?", "o que isso significa?" e "como solucionar?". Um método adequado de avaliar o comportamento é através de todo o rebanho e aos poucos reduzir o número de animais a se analisar, e por fim observar cada indivíduo, e então, comparar se é uma alteração isolada ou uma alteração que virá a ocorrer em mais animais da propriedade (Hulsen, 2016).

Embora os sinais de dor nos ruminantes possam não ser fáceis de reconhecer, as alterações associadas à dor geralmente podem ser vistas na aparência, postura, claudicação, apetite, peso, interação com outros animais e com o meio ambiente, na frequência de movimento e vocalização (Hudson et al., 2008, Chapinal et al., 2010). Além disso, os animais com dor podem proteger, lamber ou morder a área da ferida (Millman, 2013).

Com objetivo de avaliar a dor nos animais, estudos vêm sendo desenvolvidos para validar técnicas. Uma delas é desenvolvendo escalas observacionais para classificar e quantificar o grau de dor, específicos para cada espécie e conforme a origem da dor, no qual torna algumas alterações comportamentais mais específicas. São vários os tipos de escalas existentes, por exemplo, escala facial ou ortopédica, de forma visual ou numérica.

Muller et al. (2014) avaliou a expressão facial ou unidades de ação (UA) de bovinos de corte submetidos ao manejo de marcação com ferro quente, como resultado ao estimulo agudo de dor as UA apresentaram orelhas para trás, narina dilatada, abertura de boca e elevação medial e lateral da sobrancelha. Gleerup et al. (2015), observou em vacas leiteiras alterações tais como: atenção ao ambiente, posição da cabeça, posição das orelhas, expressões faciais, resposta à aproximação e posição das costas. Os mesmos sugerem que a escala de dor da vaca pode ser usada para identificar vacas com dor. Dados como esses contribuem para o desenvolvimento de métodos de diagnóstico de sinais álgicos em ruminantes. Dalla Costa (2014) avaliou a dor associada à castração em equinos, o estudo também identificou mudanças nas expressões faciais nos animais que foram submetidos à castração, o grupo controle que não obteve tratamento analgésico apresentou alterações como mudança na posição da orelha, aperto orbital e tensão nos músculos da mastigação.

De acordo com Muir \& Gaynor (2009), animas acometidos com afecções podais apresentam comportamentos de dor como preservação do membro lesionado, distribuição anormal do peso, membros apoiados na ponta, suspensos ou rotacionados, movimentos anormais, relutância para se mover e dorso arqueado. Silva et al. (2006) traz que as podopatias refletem negativamente no desempenho dos animais, de modo que animais afetados se locomovem menos no ambiente em que estão inseridos, em consequência o acesso a praça de alimentação, bebedouros e pasto se torna limitado, comprometendo assim a nutrição e a saúde do rebanho. Estudo mais recente, realizado por Barbosa et al. (2018) avaliando métodos observacionais subjetivos, como escore de locomoção (EL) e escore de condição corporal (ECC), amplamente utilizados para avaliação populacional de diferentes graus de claudicação e estado nutricional em bovinos, observaram correlação negativa entre o ECC e o EL em vacas leiteiras submetidas ao regime alimentar semiextensivo. Práticas como essas inseridas na rotina das propriedades rurais tem o potencial de possibilitar a visualização da oscilação do status metabólico do rebanho e suas possíveis causas e/ou consequências ao sistema locomotor de bovinos ao longo do tempo, podendo-se assim tomar medidas de controle e profilaxia.

Além dessas, existem diversas outras escalas de observação, com o mesmo propósito, porém variam conforme finalidade, existem escores para avaliar o estado nutricional dos animais e transtornos metabólicos, tais como escore ruminal, escore de fezes e ECC. Ainda existem os escore de higiene e escore de tetos, os quais demonstram além de problemas com as vacas, os problemas associados a fazenda, sejam elas instalações, logística, manejo e limpeza das instalações (Hulsen, 2016). 
Viñuela-Fernández et al. (2007), com objetivo de superar as deficiências das escalas de observação, desenvolveram uma escala interativa, onde o efeito verbal, interação física e/ou palpação suave da região afetada ou local da cirurgia se faz necessária. Essa escala abrange uma contagem variável com o objetivo de avaliar as alterações fisiológicas e comportamentais dos animais. É importante o observador estar familiarizado com o comportamento do animal para poder validar algumas respostas obtidas (Teixeira, 2005). Devido à subjetividade, essas escalas devem ser usadas com cuidado na prática veterinária, pois existem comportamentos diferenciados entre as espécies animais (Viñuela-Fernández et al., 2007).

Afim de contribuir com dados comportamentais, avaliações fisiológicas como frequência cardíaca e a variabilidade do batimento cardíaco podem ajudar na avaliação e interpretação de respostas como dor crônica e de curto prazo, afim de fornecer informações sobre as condições patológicas dos bovinos (Kovács et al.,2014). Tadich et al. (2013) ao avaliar frequência cardíaca e respiratória e a temperatura retal de vacas com diferentes escores de locomoção (EL), observaram alteração maiores apenas em vacas com EL 5, sugerindo que essas medidas são insensíveis à dor ou ao estresse. Ou seja, esses indicadores quando avaliados sozinhos se mostrou inaplicável. Em contrapartida, a frequência cardíaca foi elevada em estudo realizado com equinos pós-cirurgia de celiotomia, no entanto não houve influência na frequência respiratória avaliada pelos autores (Pritchett et al., 2003).

A concentração de cortisol tem sido amplamente utilizada como parâmetro para medir o estresse associado à dor e desconforto em bovinos (Coetzee, 2011). Ballou et al. (2013) e Sutherland et al. (2013) observaram um aumento nas concentrações plasmáticas de cortisol quando combinado castração com descorna. Burnett et al. (2015) ao avaliar vacas acometidas com endometrite clínica relataram maiores concentrações de cortisol quando comparados aos animais clinicamente saudáveis. Corroborando, em equinos também foi observado aumento nos níveis de cortisol sérico (Pritchett et al., 2003).

Outros biomarcadores também são utilizados para mensurar a dor nos animais. Huzzey et al. (2009) agrupando os animais em metrite grave e leve e animais saudáveis, e ao analisar concentração de haptoglobina (Hp) no pré e pós parto de vacas holandesas, obtiveram maiores concentrações de $\mathrm{Hp}$ nos animais com metrite grave e leve comparado a vacas saudáveis. Assim como Barragan et al. (2018), que também relataram aumento nas concentrações de Hp, bem como maior concentração circulante da substância P em vacas com metrite. Este último demonstrando ser um importante biomarcador de dor e inflamação (Van Engen et al. 2014; Barragan et al. 2018). Dessa forma, essas observações e biomarcadores contribuem para o diagnóstico precoce de possíveis complicações.

\section{INFLUÊNCIA DA DOR NO COMPORTAMENTO SOCIAL}

A dor é um importante aspecto que afeta negativamente o BEA como já mencionado, principalmente nos bovinos (Huxley \& Whay, 2006; Fajt et al. 2011). Al- terações como reduzir atividade, comportamento alimentar, interações sociais e aumentar o tempo em ócio são comuns em vacas nessa situação. Esse conjunto de fatores são comportamentos anormais chamados por alguns autores de "comportamentos de doença" (Harden et al., 2015; Perier et al., 2019), que é definido como uma resposta comportamental adaptativa à infecção ou lesão que apresenta características estereotipadas e é causada pelo sistema nervoso central e imunológico (Johnson, 2002; Tizard, 2008). De acordo com Tizard (2008) a maioria dessas alterações comportamentais adaptativas está ligada à depressão, perda de apetite e perda inexplicada de peso, bem como, à dor (Dittrich et al., 2019).

Tais escalas, entretanto, apresentam limitações importantes como a influência do tempo de observação e a experiência do observador, especialmente em situações de dor leve a moderada (Lindegaard et al., 2010; Sutton et al., 2013). E a avaliação do comportamento animal, em muitas propriedades, depende de uma identificação subjetiva pelos funcionários da fazenda (González et al., 2008). Conforme Barkema et al. (2015) e Dittrich et al. (2019), essa identificação será ainda mais precária, devido ao número crescente de vacas nos rebanhos e a menor interação entre humanos e animais ao longo dos anos tornando difícil a utilização de ferramentas para monitorar as alterações nos padrões comportamentais e, consequentemente, o estado de saúde de vacas (Weary et al., 2009). Porém a implementação de tais tecnologias por meio de programas controlados por computador pode se tornar um instrumento valioso, a fim de melhorar o bem-estar animal e reduzir as perdas de produção (González et al., 2008).

Estas tecnologias são desenvolvidas através de sistemas de sensores que podem ser usados para medir padrões comportamentais como atividade, ócio, alimentação e ruminação. Os padrões comportamentais mudam com a ocorrência da doença, mas também interagem com fatores externos, por mudanças climáticas e estro (Weary et al., 2009). Alterações como duração prolongada de ócio ou duração reduzida da alimentação causada por distúrbios metabólicos ou infecções, servem como ferramenta de detecção para indivíduos doentes (Dittrich et al., 2019). Dessa forma, existe um enorme potencial para muitas dessas tecnologias melhorarem a saúde, o bem-estar e o desempenho reprodutivo, pois essas ferramentas são objetivas e têm sido relatadas com frequência para identificar animais que precisam de atenção em tempo hábil (Rutten et al., 2013; Chanvallon et al., 2014).

As principais partes dos padrões de comportamento são ócio e atividade física. O ócio pode ser subdividido em deitado e em pé; a atividade física é dividida em atividade diária de caminhada, aumento da atividade durante o estro e inquietação na ordenha (Dittrich et al., 2019). A resposta comportamental mais destacada à doença (Sepúlveda-Varas et al., 2016; Itle et al., 2015) e a dor (Molgaard et al., 2012) é o maior tempo em ócio, a qual é reduzida por citocinas inflamatórias (Dittrich et al., 2019), sendo típico quando os mamíferos adoecem com uma doença infecciosa, pois o sistema imunológico requer conservação de energia para ajudar o animal a se recuperar (Johnson, 2002). A redução da atividade 
física, acontece devido a uma lesão e/ou a dor associada (Broom, 2006; Proudfoot et al., 2014).

Dentre as atividades, as vacas têm uma forte motivação para descansar (Hulsen, 2016), sendo o primeiro comportamento alterado ao apresentar algum desconforto ou dor (Whay \& Shearer, 2017). No entanto, essa diferença comportamental varia de acordo com a enfermidade e ambiente. Por exemplo, vários estudos descobriram que vacas com mastite passam mais tempo em pé quando estão doentes, evitando pressão dolorosa no úbere inflamado (Siivonen et al., 2011; Fogsgaard et al., 2012; Roches et al., 2017). Barragan et al. (2018) identificaram que vacas com metrite tenderam a passar mais tempo deitadas do que as vacas saudáveis, no entanto, a metrite também está associada à dor visceral (Stojkov et al., 2015), possivelmente resultando em evitar deitar ou dificultar o movimento de deitar. Já o comportamento de vacas claudicantes é influenciado pela severidade da claudicação, pelas instalações, pelos níveis de temperatura e umidade do ambiente e pela suavidade das superfícies em que deitam (Gomez \& Cook, 2010).

As vacas leiteiras gastam em média metade do tempo diário deitadas (Munksgaard et al., 2005 ; Gomez \& Cook, 2010). Quando são privadas de deitar e aumenta o tempo em pé no estábulo, resultam em maior número de lesões nos cascos e incidência de claudicação (Gomez \& Cook, 2010). Lesões nos cascos ou extremidades afetam diretamente o sistema locomotor das vacas, causando mudanças visíveis nos padrões de marcha, mesmo em vacas moderadamente mancas (Barragan et al., 2018). De tal maneira que o tempo em ócio dessas vacas aumenta ainda mais em comparação com as vacas sem lesões, visto que aumentam a duração de suas sessões em ócio, possivelmente para que gastem menos tempo em pé e tenham que passar pela transição de deitar e ficar em pé menos vezes (Whay \& Shearer, 2017; Weigele et al., 2018).

Um estudo realizado comparando o comportamento de vacas com e sem metrite, observaram que durante as duas semanas antes do parto, as vacas posteriormente diagnosticadas com metrite reduziram o tempo e os episódios deitadas e, três dias anteriores ao diagnóstico clínico apresentaram mais tempo com menos episódios deitadas em comparação com vacas saudáveis. Os mesmos autores sugerem que as vacas podem estar economizando energia ao se deitarem por mais tempo, resultando assim em menos episódios ao longo do dia (Neave et al., 2018). Colaborando com essa informação, Barragan et al. (2018) identificaram uma associação entre metrite clínica e tempo diário em ócio de vacas primíparas, no qual vacas diagnosticadas com metrite passaram mais tempo deitadas em comparação com as vacas sem metrite, porém não encontram o mesmo em vacas multíparas. Ainda nesse estudo, no geral as vacas do grupo com metrite tenderam a passar mais tempo deitadas do que as vacas saudáveis. A metrite clínica pode causar sinais sistêmicos associados à doença, como depressão e diminuição do apetite em vacas leiteiras (Sheldon et al., 2006), o que pode explicar a tendência desses animais de passarem mais tempo deitados (Barragan et al., 2018).
Mudanças no comportamento de ócio antes do diagnóstico também foram encontradas em vacas com cetose clínica, no qual passaram menos tempo deitado na semana que antecede ao parto e tiveram menos atividade no dia do parto (Itle et al., 2015). Em contrapartida, Kaufman et al. (2016) não relataram diferenças no tempo de ócio, número ou duração de sessão em ócio antes do diagnóstico de cetose. Vacas com mastite também apresentaram diminuição do tempo em ócio, possivelmente em reação à pressão dolorosa no úbere ao mentir (Fogsgaard et al., 2012; Roches et al., 2017).

Contudo, Proudfoot et al. (2014) demonstraram uma ligação entre o aparecimento da doença e o ócio, ocorrendo aumento da duração do ócio para vacas doentes em comparação com vacas saudáveis no período pós-parto precoce. Destacando assim, que o aumento no tempo de ócio, causa redução na produtividade, aumenta o número de problemas de saúde e redução do BEA (Munksgaard et al., 2005; Schirmann et al., 2012).

\section{AlteraÇÕES E AVAliAÇÕES dO COMPORTAMENTO AlimENTAR}

Como já mencionado, o padrão de ruminação e comportamento alimentar desempenham papéis importantes na saúde, produtividade e bem-estar das vacas (Weary et al., 2009). A disponibilidade de novas tecnologias para registrar automaticamente o consumo e comportamento alimentar estão cada vez mais disponíveis nas propriedades leiteiras e, estão demostrando ser indicadores valiosos para detectar risco de doenças ou lesões permitindo aumentar o uso de medidas preventivas e objetivas conforme cada caso (Weary et al., 2009).

Os movimentos da mandíbula das vacas podem ser medidos com precisão usando vários sensores (Watt et al., 2015) com saídas gravadas manualmente ou resumidas usando software para interpretar o sinal para determinar a alimentação e a ruminação (Beauchemin, 2018).

De acordo com Dittrich et al. (2019), alguns sistemas usam um acelerômetro instalado em um brinco ou colar no pescoço para detectar movimento. Outros usam um microfone montado em um colar para detectar o som. E ainda existem os alimentadores automatizados que fornecem dados de consumo, taxa de alimentação, tempo de alimentação, tamanho de refeição e visitas aos alimentadores. Todos esses sistemas fornecem aos funcionários uma avaliação rápida e não perturbam o comportamento natural do animal, favorecendo a utilização dessas tecnologias para tomadas de decisões dos funcionários das propriedades (Beauchemin, 2018; Dittrich et al., 2019).

A alimentação reduzida é comumente usada como um indicador de doença e pode ser vista mesmo antes que os sintomas clínicos da doença apareçam (Weary et al., 2009). Esta redução pode ser motivada por vários mecanismos diferentes, incluindo baixa motivação para procurar comida devido à perda de apetite. Além disso, animais doentes podem conservar energia permanecendo deitados ao invés de gastar energia procurando alimentos (Hart, 1988). As vacas doentes também podem limitar as visitas aos alimentadores 
para evitar encontros agressivos que possam ser necessários para ingerir alimentos frescos (Huzzey et al., 2006), ou podem optar por permanecer deitadas devido ao aumento da motivação para descansar ou dormir, o que pode combater os sentimentos de mal-estar (Millman, 2007).

Dessa maneira, a ruminação e a alimentação podem oferecer informações únicas sobre o estado geral de saúde de vacas que não são fornecidas pela avaliação e exame clínico (Stangaferro et al., 2016; Beauchemin, 2018). Lesões nos cascos e claudicação estão relacionadas com o tempo de ócio e o aumento do tempo em pé de vacas leiteiras (Gomez \& Cook, 2010) que por sua vez, alteram o comportamento alimentar, reduzindo o tempo de alimentação e aumentando a taxa de alimentação e o tamanho da refeição, o que pode resultar em aumento da produção de ácido ruminal e distúrbios digestivos (Munksgaard et al., 2005).

Sepúlveda-Varas et al. (2016) relataram mudanças no comportamento alimentar nos dias anteriores ao diagnóstico de vacas com mastite, havendo redução no consumo de ração nos dias antes do diagnóstico, ocorrendo queda na taxa de alimentação sem influenciar o tempo de alimentação.

Em relação ao comportamento alimentar, a metrite foi associada a uma diminuição na ingestão de matéria seca (IMS) no pré-parto de vacas leiteiras. Huzzey et al. (2007) mostraram diferença na IMS pré-parto e no comportamento alimentar em vacas que desenvolveram metrite clínica após o parto. Um estudo mais recente demonstrou que as vacas diagnosticadas com metrite comiam cerca de $1 \mathrm{~kg}$ a menos por dia e realizavam menos refeições durante os 3 dias anteriores ao diagnóstico, havendo maior diferença nas refeições quando as vacas foram diagnosticadas perto do parto (Neave et al., 2018). Pérez-Baez et al. (2019) observaram que vacas com metrite apresentaram menor porcentagem de IMS do peso corporal no pré-parto, bem como balanço energético. Cada redução de 0,1 ponto percentual na IMS em peso médio e cada 1-Mcal no balanço energético médio, nos últimos 3 dias pré-parto, aumentaram as chances de ter metrite em $8 \%$.

A IMS e o tempo de alimentação ainda variam conforme o grau de gravidade da metrite, havendo menor IMS e tempo de alimentação em vacas gravemente doentes (escore 4 com febre) em comparação com vacas levemente doentes (escore 2 ou 3, com ou sem febre) (Huzzey et al., 2007; Urton et al., 2005). No entanto, existem estudos contraditórios, no qual não evidenciaram diferenças na IMS e no comportamento alimentar entre vacas no pré-parto posteriormente diagnosticadas com metrite e vacas que permaneceram saudáveis (Schirmann et al., 2016).

Liboreiro et al. (2015) relataram que vacas diagnosticadas com metrite clínica passaram menos tempo ruminando e tiveram atividade reduzida em comparação com vacas sem metrite clínica. Neave et al. (2018) não encontraram diferença significativa no número de visitas aos alimentadores antes dos sintomas clínicos de metrite, quando comparado a vacas saudáveis. Entretanto, vacas diagnosticadas com metrite e cetose no estudo, reduziram as visitas aos alimentadores, sugerindo assim, que a combinação dessas duas enfermidades pode resultar em maior mal-estar. Além disso, os mesmos autores relataram que vacas com metrite foram substituídas com mais frequência nos alimentadores, demonstrando competição por estes animais pelos alimentadores, podendo estes terem sido incapazes ou por não quererem manter seu lugar nos cochos quando deparados a competição na área de alimentação.

As diferentes maneiras pelas quais os comportamentos alimentares são afetados pelos transtornos de saúde da vaca em transição foram discutidos por González et al. (2008), que mostraram que vacas com cetose apresentaram alterações no tempo de alimentação, visitas ao cocho e taxa de alimentação, enquanto vacas mancas aumentaram a taxa de alimentação e vacas com mastite apresentaram grande variação em suas respostas comportamentais.

\section{CONCLUSÃO}

O bem-estar animal (BEA) é uma prática que deve ser adotada em qualquer sistema de produção, visto que se configura como uma demanda crescente do mercado consumidor e apresenta uma relação positiva com produtividade dos rebanhos leiteiros. Os métodos de avaliação e mensuração da dor e, consequente, de bem-estar animal são ferramentas importantes na mensuração destes marcadores mostrando-se como métodos válidos e positivos na rotina das propriedades. No entanto, ainda faltam estudos que validem técnicas observacionais, principalmente especificas para cada espécie.

Contudo, os avanços nesse quesito são constantes e têm sido realizados através da avaliação comportamental, sejam elas através de escores de dor ou observações afim de promover qualidade e segurança dos produtos e consequentemente melhorar a produtividade, sustentabilidade e lucratividade do sistema produtivo.

\section{BIBLIOGRAFIA}

Aleixo, G.A.S.; Tudury, E.A.; Coelho, M.C.O.C.; Andrade, L.S.S.; Bessa, A.L.N.G. 2016. Tratamento da dor em pequenos animais: fisiopatologia e reconhecimento da dor (revisão de literatura: parte I). Medicina Veterinária (UFRPE), Recife, 10(1-4):19-24.

Almeida T.P., et al. 2006. Classificação dos Processos Dolorosos em Medicina Veterinária. Veterinária em Foco, 3(2): 107-118.

Amaral, J.B.; Pires, R.M.L.; Ambrósio, L.A.; de Oliveira, F.A.; Trevisan, G. 2017. Expressão facial, vocalização e posturas anômalas em bovinos submetidos à eletroejaculação convencional. PUBVET, $11(11): 1085-1097$.

Anil SS, Anil L, Deen J: 2002. Challenges of pain assessment in domestic animals. J Am Vet Med Assoc., 220 (3): 313-319. 10.2460/ javma.2002.220.313.

Ballou MA, Sutherland MA, Brooks TA, Hulbert LE, Davis BL, Cobb CJ, 2013. A administração de anestésico e analgésico impede a supressão de muitas respostas leucocitárias após castração cirúrgica e descoramento físico. Veterinario. Immunol. Immunopathol; 151 (23270586): 285-29

Barbosa, A., Pizoni, C., Luz, G.B., Correa, M.N., Rabassa, V.R., Pino, F.A.B. Del, Moraes, F.P., \& Martins, C.F. 2018. Relação entre escore de condição corporal e escore de locomoção em vacas leiteiras submetidas a manejo semiextensivo de alimentação. Arquivo Brasileiro 
de Medicina Veterinária e Zootecnia, 70(4), 1129-1134. https:// dx.doi.org/10.1590/1678-4162-9978

Barker, Z.E., Vazquez Diosdado, J.A., Codling, E.A., Bell, N.J., Hodges, H.R., Croft, D.P., Amory, J.R., 2018. Use of novel sensors combining local positioning and acceleration to measure feeding behavior differences associated with lameness in dairy cattle. J. Dairy Sci. 101: 6310-6321.

Barkema, H.W.; Von Keyserlingk, M.A.G.; Kastelic, J.P.; LeBlanc, S.J.; Keefe, G.P.; Kelton, D.F. 2015. Invited review: Changes in the dairy industry affecting dairy cattle health and welfare. J. dairy science, 98(1 1):7426-7445, Doi: https://doi.org/10.3168/jds.2015-9377

Barragan A. A., Piñeiro J. M., Schuenemann G. M., Rajala-Schultz P. J., Sanders D. E., Lakritz J., e Bas S., 2018. Evaluation of daily activity patterns and biomarkers of pain, inflammation and stress in lactating dairy cows diagnosed with clinical metritis. Journal Dairy Science, 101:8248-8258. doi: 10.3168/jds.2018-14510.

Beauchemin, K.A. 2018. Invited review: Current perspectives on eating and rumination activity in dairy cows. Journal Dairy Science. 101(6):4762-4784

Bond, G. B.; Almeida, R.; Ostrensky, A. M., Maiolino, C. F. 2012. Métodos de diagnóstico e pontos críticos de bem-estar de bovinos leiteiros. Ciência Rural, 42: 1286-1293. https://doi.org/10.1590/ S0103-84782012005000044

Broom, D.M., Fraser, A.F., 2015. Domestic Animal Behaviour and Welfare, fifth ed. CABI, Wallingford, Boston.

Broom, D.M., Molento, C.F.M. 2004. Bem-estar animal: conceito e questões relacionadas - Revisão. Archives of Veterinary Science, 9(2): 1-11.

Bruijnis, M. R. N.; Hogeveen, H. and Stassen, E. N. 2013. Measures to improve dairy cow foot health: consequences for farmer income and dairy cow welfare. Animal, 7:1, pp 167-175.

Burnett, T.A., Madureira, A.M.L., Silper, B.F., Tahmasbi, A., Nadalin, A., Veira, D.M., Cerri, R.L.A. 2015. Relationship of concentrations of cortisol in hair with health, biomarkers in blood, and reproductive status in dairy cows. J. Dairy Science, 98: $4414-4426$. https://doi. org/10.3168/jds.2014-8871

Cimmino MA, Ferrone C, Cutolo M. 201 1. Epidemiologia da dor musculoesquelética crônica. Best Pract Res Clin Rheumatol, 25 (2): 173-83.

Chanvallon A. Coyral-Castel S. Gatien J. Lamy J.M. Ribaud D. Allain C. Clément P. Salvetti P. 2014. Comparison of three devices for the automated detection of estrus in dairy cows. Theriogenology, 82: 734-741.

Chapinal, N., de Passille, A.M., Rushen, J., Wagner, S., 2010. Automated methods for detecting lameness and measuring analgesia in dairy cattle. J. Dairy Sci. 93, 2007-2013.

Coetzee, J. F. 2011. A review of pain assessment techniques and pharmacological approaches to pain relief after bovine castration: practical implications for cattle production within the United States. Appl. Anim. Behav. Sci. 135:192-213.

Coetzee, J.F.; Shearer, J.K.; Stock, M.L.; Kleinhenz, M.D.; Amstel, S.R.V. 2017. An Update on the Assessment and Management of Pain Associated with Lameness in Cattle. Vet Clin Food Animal, 33: 389-41 1.

Dalla Costa, E. et al. 2014. Development of the Horse Grimace Scale (HGS) as a pain assessment tool in horses undergoing routine castration. PLoS ONE, 9(3).

Des Roches, A.D.B., Faure, M., Lussert, A., Herry, V., Rainard, P., Durand, D., Foucras, G., 2017. Behavioral and patho-physiological response as possible signs of pain in dairy cows during Escherichia coli mastitis. A pilot study. J Dairy Science, 100: 8385-8397.

Dittrich, I.; Gertz, M.; Krieter, J. 2019. Alterations in sick dairy cows' daily behavioural patterns. Heliyon, 5(11).

Erickson, H. H., \& Kitchell, R. L. 1992. Fed Proc. In_Nicolas H. Booth, Farmacologia e Terapêutica em

Veterinária. 6.ed. Rio de Janeiro: Guanabara Koogan.

Fajł VR, Wagner SA, Norby B. 2011 . Analgesic drug administration and attitudes about analgesia in cattle among bovine practitioners in the United States. J Am Vet Med Assoc, 238:755-67.

Farm Animal Welfare Council. 1993. Report on priorities for animal welfare, research and development. London: Farm Animal Welfare Council.
Flecknell, P. 2008. Analgesia from a veterinary perspective. BritJ Anaest, 101(1): 121-124. Doi: 10.1093/bja/aen087.

Flower, F. C. and Weary, D. M. 2006. Effect of Hoof Pathologies on Subjective Assessments of Dairy Cow Gait. J. Dairy Science, 89:139-146.

Fogsgaard, K.K., Røntved, C.M., Sørensen, P., Herskin, M.S., 2012. Sickness behavior in dairy cows during Escherichia coli mastitis. J. Dairy Science. 95: 630-638.

Gleerup, K.B.; Andersen, P.H.; Munksgaard, L.; Forkman, B. 2015. Pain evaluation in dairy cattle. Applied Animal Behaviour Science, 171: 25-32.

Gomez A, Cook NB. 2010. Time budgets of lactating dairy cattle in commercial freestall herds. Journal Dairy Science, 93:5772-5781.

González, L.A.; Tolkamp, B.J.; Coey, M.P.; Ferret, A.; Kyriazakis, I. 2008. Changes in feeding behaviour as possible indicators for the automatic monitoring of health disorders in dairy cows. J. Dairy Sci., 91: 1017-1028

Gruber, S.; Mansfeld, R. 2019. Herd health monitoring in dairy farms - discover metabolic diseases. An overview. Gesundheitsmonitoring in Milchviehherden - Stoffwechselstörungen rechtzeitig erkennen. Ein Überblick. Tierarzt Prax Ausg G Grosstiere Nutztiere. 47(4):246-255. doi:10.1055/a-0949-1637

Harden, L.M., Kent, S., Pittman, Q.J., Roth, J., 2015. Fever and sickness behavior. Friend or foe? Brain Behav. Immun. 50, 322-333.

Hellebrekers, L. J. 2002. Dor em Animais. $1^{\circ}$ ed. São Paulo: Manole.

Hoag, T.M.; Lemme, C.F. 2018. Indústria de alimentos de origem animal: riscos e oportunidades para o setor decorrentes das políticas de bem-estar animal. Revista de Administração de Empresas, 58(3), 244-253. https://dx.doi.org/10.1590/s0034-759020180305

Hudson, C.; Whay, H.; Huxley, J. 2008. Recognition and management of pain in cattle. In Practice, 30: 126-134.

Huxley JN, Whay HR. 2006. Current attitudes of cattle practitioners to pain and the use of analgesics in cattle. Veterinary Record, 159: 662-668.

Huzzey, J., Duffield, T., LeBlanc, S., Veira, D., Weary, D., Von Keyserlingk, M. 2009. Short communication: haptoglobin as an early indicator of metritis. J. Dairy Science, 92: 621-625. https://doi.org/10.3168/ jids.2008-1526

IASP - Internacional Association for the Study of PainTaxonomy. 2011. Pain terms, a current list with definitions and notes on usage. $p$. 209-214. 1994. Disponível em: < www.iasp-pain.org/Content/NavigationMenu/GeneralResourcelinks/PainDefinitions/default.htm>. Acesso em: 01/07/2020.

Itle, A.J., Huzzey, J.M., Weary, D.M., Von Keyserlingk, M.A.G., 2015. Clinical ketosis and standing behavior in transition cows. J. Dairy Sci. 98, 128-134.

Johnson, R.W., 2002. The concept of sickness behavior. A brief chronological account of four key discoveries. Vet. Immunol. Immunopathol. 87:443-450.

Kaufman, E.I., LeBlanc, S.J., McBride, B.W., Duffield, T.F., DeVries, T. J. 2016. Short communication: Association of lying behavior and subclinical ketosis in transition dairy cows. J. Dairy Sci. 99:7473-7480.

Klaumann, P. P.; Wouk, A.F.P.F.; Sillas, T. 2008. Patofisiologia da dor. Archives of Veterinary Science, 13(1): 1-12.

Kovács, L., Jurkovich, V., Bakony, M., Szenci, O., Póti, P., T凶zsér, J. 2014. Welfare implication of measuring heart rate and heart rate variability in dairy cattle: literature review and conclusions for future research. Animal, 8(2):316-330. doi: 10.1017 / S1751731113002140.

Leblanc, S.J.; Lissemore, K.D.; Kelton, D.F.; Dueld, T.F.; Leslie, K.E. 2006. Major advances in disease prevention in dairy cattle. J. Dairy Sci, 89:1267-1279.

Liboreiro, D.N., Machado, K.S., Silva, P.R.B., Maturana, M.M., Nishimura, T.K., Brandão, A.P., Endres, M.I., Chebel, R.C., 2015. Characterization of peripartum rumination and activity of cows diagnosed with metabolic and uterine diseases. J. Dairy Sci., 98:6812-6827.

Lindegaard, C., Thomsen, M.H., Larsen, S., Andersen, P.H. 2010. Analgesic efficacy of intra-articular morphine in experimentally induced radiocarpal synovitis in horses. Veterinary Anaesthesia and 
Analgesia, 37(2):171-185. http://dx.doi.org/10.1111/i.1467. 2995.2009.00521.x

Millman, S.T. 2013. Behavioral Responses of Cattle to Pain and Implications for Diagnosis, Management, and Animal Welfare. Vet Clin Food Anim, 29:47-58. http://dx.doi.org/10.1016/j.cvfa.2012.11.007

Molento, C.F.M. 2005. Bem-estar e produção animal: Aspectos Econômicos - Revisão. Archives of Veterinary Science, 10(1): 1-11.

Mølgaard, L.; Damgaard, B.M.; Bjerre-Harpøth, V.; Herskin, M.S. 2012. Effects of percutaneous needle liver biopsy on dairy cow behaviour. Research in Veterinary Science, 93(3): 1248-1254.

Muir, W. W., Gaynor, J. S. 2009. Manual de controle da dor em medicina veterinária. MedVet, São Paulo, Brasil.

Müller, B.R.; Hammerschmidt, J.; Feldens, C.S.; Molento, C.F.M. 2014. Unidades faciais de ação associadas à dor em bovinos de corte. III congresso brasileiro de bioética e bem-estar animal. Senciência e Bem-estar Animal - Expandindo Horizontes, Curitiba, PR.

Munksgaard, L., Jensen, M.B., Pedersen, L.J., Hansen, S.W., Matthews, L., 2005. Quantifying behavioural priorities - effects of time constraints on behaviour of dairy cows, Bos taurus. Appl. Anim. Behav. Sci. 92, 3-14.

Neave, H.W., Lomb, J., Weary, D.M., Leblanc, S.J., Huzzey, J.M., Von Keyserlingk, M.A.G., 2018. Behavioral changes before metritis diagnosis in dairy cows. J. Dairy Sci. 101:1-12. doi.org/10.3168/ jds.2017-13078

OIE (World Organization for Animal Health). 2019. Chapter 7.1: Introduction to the recommendations for animal welfare. In: Terrestrial Animal Health Code, Volume 1. Disponível em: <http://www.oie.int/ indexhttps: $/ /$ www. oie.int/index. php? id $=169 \& \mathrm{~L}=2 \&$ htmfile=chapitre aw introduction. $h$ tm $>$ Acessado em 02/07/2020.

Palmer, M.A., Law, R., O'Connell, N.E., 2012. Relationships between lameness and feeding behaviour in cubicle-housed Holstein-Friesian dairy cows. Appl. Anim. Behav. Sci. 140, 121-127.

Pérez-Báez, J.; Risco, C.A.; Chebel, R.C.; Gomes, G.C.; Greco, L.F.; Tao, S.; Thompson, I.M.; do Amaral, B.C.; Zenobi, M.G.; Martinez, N.; Staples, C.R.; Dahl, G.E.; Hernández, J.A.; Santos, J.E.P.; Galvão, K.N. 2019. Association of dry matter intake and energy balance prepartum and postpartum with health disorders postpartum: Part I. Calving disorders and metritis. J. Dairy Sci. 102 (10). https://doi. org/10.3168/ids.2018-15878

Perier, N.; des Roches, A.B.; Jensen, M.B.; Proudfoot, K. 2019. Infectious Disease Does Not Impact the Lying and Grooming Behaviour of PostParturient Dairy Cows. Animals, 9, 634; doi:10.3390/ani9090634.

Pritchett, L.C.; Ulibarri, C.; Roberts, M.C.; Schneider, R.K.; Sellon, D.C. 2003. Identification of potential physiological and behavioral indicators of postoperative pain in horses after exploratory celiotomy for colic. Applied Animal Behaviour Science, 80(1):31-43.

Proudfoot, K.L., Jensen, M.B., Weary, D.M., Von Keyserlingk, M.A.G., 2014. Dairy cows seek isolation at calving and when ill. J. Dairy Sci., 97: 2731-2739.

Rutten, C.J.; Velthuis, A.G.J.; Steeneveld, W.; Hogeveen, H. 2013. Invited review: Sensors to support health management on dairy farms. J. Dairy Sci., 96: 1928-1952

Schirmann, K., Chapinal, N., Weary, D.M., Heuwieser, W., Von Keyserlingk, M.A.G., 2012. Rumination and its relationship to feeding and lying behavior in Holstein dairy cows. J. Dairy Sci., 95, 321 2-3217.

Schirmann, K.; Weary, D.M.; Heuwieser, W.; Chapinal, N.; Cerri, R.L.A.; Von keyserlingk, M.A.G. 2016. Short communication: Rumination and feeding behaviours dier between healthy and sick dairy cows during the transition period. J. Dairy Sci., 99, 9917-9924.

Sepúlveda-Varas, P., Proudfoot, K.L, Weary, D.M., Von Keyserlingk, M.A.G. 2016. Changes in behaviour of dairy cows with clinical mastitis. Applied Animal Behaviour Science, 175:8-13. https://doi. org/10.1016/i.applanim.2014.09.022

Sheldon, I. M., G. S. Lewis, S. LeBlanc, and R. O. Gilbert. 2006. Defining postpartum uterine disease in cattle. Theriogenology, 65:1516-1530.

Siivonen, J., Taponen, S., Hovinen, M., Pastell, M., Lensink, B.J., Py€or€al€a, S., HEanninen, L., 2011. Impact of acute clinical mastitis on cow behaviour. Appl. Anim. Behav. Sci. 132, 101-106.
Silva, L.A.F., Fioravanti, M.C.S., Silva, M.A.M.; Cunha, P.H.J., Sousa, V.R., Silva, O.C., Macedo, S.P., Moura, M.I., Garcia, A.M. 2006. Enfermidades digitais em bovinos confinados: Uso parenteral do cobre na prevenção. Vet. Not., Uberlândia, 12(1): 21-28.

Silva, S.T.G.; Tenório, A.P.M.; Afonso, J.A.B.; Carvalho, A.Q. 2011. Fisiopatologia da dor em ruminantes e equinos. Medicina Veterinária, Recife, 5(1): 18-23.

Stangaferro, M. L.;Wijma, R.; Caixeta, L. S.;Al-Abri, M. A. and Giordano, J. O. 2016. Use of rumination and activity monitoring for the identification of dairy cows with health disorders: Part III. Metritis. J. Dairy Sci. 99:7422-7433. http://dx.doi.org/10.3168/ids.2016-11352

Stojkov, J.; Von Keyserlingk, M. A. G.; J. N. Marchant-Forde, J. N.; Weary, D. M. 2015. Assessment of visceral pain associated with metritis in dairy cows. J. Dairy Science, 98:5352-5361 http://dx. doi. org/10.3168/ids.2014-9296

Sutherland MA, Ballou MA, Davis BL, Brooks TA, 2013. Efeito da castração e descornação singular ou combinada no comportamento e fisiologia de bezerros da raça Holandesa. Journal Anim. Sci., 91 (23287529): 935-942.

Sutton, G.A.; Dahan, R.; Turner, D.; Paltiel, O. 2013. A behaviour-based pain scale for horses with acute colic: Scale construction. The Veterinary Journal, 196: 394-401.

Tadich, N.; Tejeda, C.; Bastias, S.; Rosenfeld, C.; Green, L.E. 2013. Nociceptive threshold, blood constituents and physiological values in 213 cows with locomotion scores ranging from normal to severely lame. The Veterinary Journal, 197(2): 401-405.

Teixeira, M.W. 2005. Dor em pequenos animais. Revista do Conselho Federal de Medicina Veterinária, Brasília, n. 34: 31-41.

Thomsen PT, Gidekull M, Kerskin MS.2010. Scandinavian bovine practitioners' attitudes to the use of analgesics in cattle. Vet Record, 167:256-258.

Tizard, I., 2008. Sickness behavior, its mechanisms and significance. Anim. Health Res. Rev. 9, 87-99.

Trindade, H.I.; Batista, MCS.; Silva, LLB. 2013. Dor: mecanismos envolvidos na sua transmissão e recursos terapêuticos aplicados à sua inibição. Medicina Veterinária, Recife, 7(4):6-18.

Urton, G., Von Keyserlingk, M.A.G., Weary, D.M., 2005. Feeding behavior identifies dairy cows at risk for metritis. J. Dairy Sci. 88, 2843-2849.

Van Engen, N.K, Stock, M.L, Engelken, T., Vann, R.C, Wulf, L.W, Karriker, L.A, Busby, W.D, Lakritz, J., Carpenter, A.J, Bradford, B.J, Hsu, W.H, Wang, C., Coetzee, J.F. 2014. Impacto do meloxicam oral na circulação fisiológica biomarcadores de estresse e inflamação em novilhos de corte após transporte de longa distância, Journal of Animal Science, 92(2): 498-510.

Viñuela-Fernández, I. Jones E, Welsh E.M, Fleetwood-Walker S.M. 2007. Pain mechanisms and their implication for the management of pain in farm and companion animals. The Veterinary Journal, 174:227-239.

Von Keyserlingk, M.A.; Rushen, J.; de Passillé, A.M.; Weary, D.M. 2009. Invited review: The welfare of dairy cattle-Key concepts and the role of science. J. Dairy Sci., 92: 4101-4111

Von Keyserlingk, M.A.; Martin, N.P.; Kebreab, E.; Knowlton, K.F.; Grant, R.J.; Stephenson, M.; Sniffen, C.J.; Harner, J.P.; Wright, A.D.; Smith, S.I. 2013. Invited review: Sustainability of the US dairy industry. J. Dairy Sci., 96: 5405-5425

Watt, L.J.; Clark, C.E.F.; Krebs, G.L.; Petzel, C.E.; Nielsen, S.; Utsumi, S.A. 2015. Differential rumination, intake, and enteric methane production of dairy cows in a pasture-based automatic milking system. J. Dairy Sci., 98 (26254528): 7248-7263. 10.3168/jds.2015-9463

Weary, D. M.; Huzzey, J. M. and von Keyserlingk, M. A. G. 2009. Board-invited review: Using behavior to predict and identify ill health in animals. J. Anim. Sci. 87:770-777 doi:10.2527/jas.2008-1297

Weigele, H.C., Gygax, L., Steiner, A., Wechsler, B., Burla, J.-B., 2018. Moderate lameness leads to marked behavioral changes in dairy cows. J. Dairy Sci. 101, 2370-2382.

Whay, H.R.; Shearer, J.K. 2017. The Impact of Lameness on Welfare of the Dairy Cow. Veterinary Clinics Food Animal. 33(2):153-164. 